\title{
Delay to diagnosis and breast cancer stage in an urban South African breast clinic
}

\author{
S Rayne, ${ }^{1} \mathrm{MB}$ ChB, FCS (SA); K Schnippel, ${ }^{2} \mathrm{PhD}$; D Kruger, ${ }^{1} \mathrm{PhD}$; C-A Benn, ${ }^{1} \mathrm{MB}$ ChB, FCS (SA); C Firnhaber, ${ }^{3} \mathrm{MD}$ \\ ${ }^{1}$ Department of Surgery, Helen Joseph Hospital and Faculty of Health Sciences, University of the Witwatersrand, Johannesburg, South Africa \\ ${ }^{2}$ Health Economics Unit, School of Public Health and Family Medicine, Faculty of Health Sciences, University of Cape Town, South Africa \\ ${ }^{3}$ Clinical HIV Research Unit, Department of Internal Medicine, Faculty of Health Sciences, University of the Witwatersrand, Johannesburg, South Africa
}

Corresponding author: S Rayne (rayne.sarah@gmail.com)

\begin{abstract}
Background. Breast cancer is the most common cancer in women in many low- and middle-income countries, and often presents at an advanced stage that affects prognosis irrespective of the care available. Although patient-related delay is commonly cited, the reasons for delay and the relationship of delay to stage are still poorly documented, especially in Africa.

Objectives. To identify where patient-related socioeconomic delays occur and how these relate to stage at presentation.

Methods. Consecutive women with a new breast cancer diagnosis were prospectively invited to complete a questionnaire on their socioeconomic characteristics and ability to access care. Clinical stage at presentation was documented.

Results. Over 14 months, 252 women completed the questionnaire (response rate $71.6 \%$ ). Their median age was 55 years (interquartile range 44 - 65), with $26.5 \%$ aged $<45$ years. Stage at presentation was stage 1 in $15.5 \%$ of patients, stage 2 in $28.5 \%$ and stage 3 in $56.0 \%$. Almost a third of the patients (30.4\%) presented with a T4 tumour (6.1\% inflammatory). Total delay in presenting to the breast clinic was significantly associated with locally advanced stage at presentation $(p=0.021)$. Average delay differed between early stage $(1.5$ months $)$ and locally advanced ( 2.5 months), and most delay occurred between acknowledging a breast symptom and seeking care. The least delay was between attending a health service and presenting at the open-access breast clinic, with $75.0 \%$ presenting within 1 month. Factors associated with delay were difficulties with transport, low level of education and fear of missing appointments due to work.

Conclusions. Most women delayed in seeking breast care. Facilitating direct access to specialist breast clinics may reduce delays in presentation and improve time to diagnosis and care.
\end{abstract}

S Afr Med J 2019;109(3):159-163. DOI:10.7196/SAMJ.2019.v109i3.13283

Breast cancer is the most common cancer in women in most lowand middle-income countries (LMICs), including South Africa (SA), where it is the most common cancer histologically diagnosed in women, with a lifetime risk of 1 in $26^{[1]}$ and causing at least $0.9 \%$ of all deaths in 2015. ${ }^{[2]}$ Although breast cancer has a good overall prognosis in comparison with many other cancers, with an $84-89 \%$ survival rate at 5 years reported in high-income countries, in LMICs these rates are substantially lower and the majority of women diagnosed with breast cancer will succumb to the disease. Mortality rates at 5 years of $47 \%$ in SA and $86.4 \%$ in Mali have been reported. ${ }^{[3]}$ This high diagnosis-to-mortality ratio could be attributable to late stage at presentation, as reported in most literature from LMICs, which would affect prognosis irrespective of the care available.

In SA, the National Department of Health recently launched a national Breast Cancer Prevention and Control Policy ${ }^{[4]}$ outlining standards to which healthcare providers should adhere when providing care. Many of the standards and much of the publicity around the policy have focused on cancer care provision through better therapeutic modalities, such as access to chemotherapy and novel monoclonal antibody therapies. ${ }^{[5]}$ However, in addition to provision of breast cancer care at tertiary services, ensuring access to early diagnosis and recognition of disease are of equal if not more importance. Where resources are limited, many expensive modalities of care may be avoided with prompt presentation and early diagnosis.

While small studies from various parts of the world suggest that patient-related delay to care is common, ${ }^{[6-10]}$ the characteristics of delay and its relationship to stage of breast cancer presentation are still poorly documented, especially in southern Africa. Older studies have cited fear of mastectomy and adherence to traditional healing as causes of delay, ${ }^{[7,11,12]}$ but more recent studies and systematic reviews show extremes of age (the youngest and oldest patients being most affected), marital status, education and income as being of most importance. ${ }^{[13]}$

\section{Objectives}

SA is a middle-income country where open-access tertiary hospitals can provide care, but deep-rooted inequities may prevent access to it by all the population. This study was designed to define the relationship between socioeconomic characteristics of patients seeking care at a tertiary specialist clinic and to describe where patient-related delays occur and how these relate to stage at presentation, which ultimately affects treatment requirements and survival.

\section{Methods}

This study was conducted in one of the few specialist breast-care clinics in SA where an open-access system allows all patients to attend without previous appointment or referral for breast examination and investigation. The format of this clinic and the spectrum of disease have been described previously, ${ }^{[14]}$ and the clinic currently diagnoses and manages $>300$ new diagnoses of breast cancer per year.

Over a 14-month period from January 2016 to February 2017, all patients who received a new breast cancer diagnosis were approached by a clinician during their post-result counselling and asked if they would participate in the study. After written consent had been 
obtained, they were asked questions from, or completed themselves, a previously piloted questionnaire regarding their demographics, socioeconomic situation, education and self-described ability to access care ${ }^{[15]}$ Participants were asked to quantify how long it took them to acknowledge or disclose a breast symptom once noticed, from that point how long before they visited any health facility, and from that point how long before they attended the open-access breast-care clinic for diagnosis. The language of the questionnaire was English, although each of two research assistants involved in participant recruitment were fluent in at least two languages, and assistance was sought from other staff members to allow participants to complete the questionnaire in a language most comfortable for them, through immediate verbal translation. The results of the questionnaire were linked via a study number to the patient's clinical stage at presentation, as documented by the diagnosing breast specialist surgeon. Ethical approval was received from the Human Research Ethics Committee of the University of the Witwatersrand, Johannesburg (ref. no. M111121).

The data were collected using REDCap (hosted by the University of the Witwatersrand) to enter and manage the data, ${ }^{[16]}$ and all statistical analyses were done in Stata version 13.1 (StataCorp, USA). Patient characteristics were described using frequencies and proportions, and age was categorised as $<45$ years or $\geq 45$ years. Marital status was categorised as either married or unmarried, the latter including participants who were single, divorced or widowed. Education was categorised according to the highest level of formal schooling obtained: completed primary schooling, completed secondary schooling, or matriculation from school with or without qualifications above that.

Participants who indicated that they had at least some difficulty with transportation or taking time off from work were compared with those who did not. Fear regarding missing an appointment or required attendance at the hospital due to work, money or transport problems was indicated on a four-category summative scale (from strongly agree to strongly disagree) and those who expressed some fear (agree or strongly agree) were compared with those who did not express any fear. Time to presentation comprised time taken to acknowledge symptom(s), time to seek healthcare services, and time taken to attend a breast-care clinic. To facilitate responses, participants were offered categories of $<1$ week, 1 week - 1 month, 1 - 6 months, 6 - 12 months, or $>1$ year. To facilitate summation and analysis of the total delay numerically, these were then converted into total days of delay, which were created by taking the minimum time for each response, e.g. $<1$ week $=1$ day, 1 week -1 month $=8$ days, 1 month -6 months $=31$ days, 6 months -1 year $=182$ days and $>1$ year $=366$ days.

Clinical stage at presentation was described using the TNM classification (8th edition), ${ }^{[17]}$ and stage was determined. Stage was then categorised into early (stage 1 - stage $2 \mathrm{a}$ ) and locally advanced (stage $2 \mathrm{~b}$ - stage $3 \mathrm{c}$ ). For determination of delay by stage, patients with stage 0 were excluded because of the relationship with screening rather than clinically detected disease in this group. Stage 4 was also excluded because these patients commonly follow a route to medical oncological care that may bypass a surgical clinic. Two-sided differences of proportions were compared using Pearson's $\chi^{2}$ test. Categorised delay was compared using ordinal logit. A $p$-value $\leq 0.05$ was considered statistically significant.

\section{Results}

Over 14 months from January 2016 to February 2017, 352 patients received a new diagnosis of breast cancer. A total of 252 patients with newly diagnosed stage $1-3$ breast cancer completed the questionnaire, giving a response rate of $71.6 \%$. Their median age was 55 years (interquartile range (IQR) 44 - 65), with 59 (26.5\%) aged $<45$ years. Stage was recorded for 232 patients $(92.1 \%)$, with stage at presentation stage 1 in $36(15.5 \%)$, stage 2 in $66(28.5 \%)$ and stage 3 in $130(56.0 \%)$. Nearly one-third of all patients presented with T4 cancer (either T4d inflammatory (6.1\%) or involving skin above or muscle below (24.3\%)). When stage was categorised, it was found that only $32.3 \%$ of patients presented with early-stage breast cancer.

Key demographics, presented according to early or advanced stage at diagnosis, are set out in Table 1. There were very few relationships between any patient-reported fears or socioeconomic characteristics and stage of disease, but self-reported transport difficulties $(p=0.011)$ and having a cell phone (mobile phone) contract $(p=0.035)$ were significantly associated with locally advanced disease at presentation. Total delay in presenting at the breast clinic was statistically associated with locally advanced stage at presentation $(p=0.021)$.

Self-reported median delay to presentation at a breast unit was 2 months (IQR 1 - 7) after noticing a symptom. Total delay differed according to whether patients were diagnosed with early-stage disease $(1.5$ months, IQR $<1-6)$ or disease at a locally advanced stage (2.5 months, IQR 1 - 7). When the data on this delay were separated out and analysed, most of the delay occurred during the period between a woman acknowledging a problem and attending a health service. This delay was a median of 1 week (IQR 1 day - 1 month) in early-stage and 1 month (IQR 1 day - 3 months) in locally advanced breast cancer. The least delay was between attending a health service and presenting at the specialist breast-care clinic, with a median of 1 week (IQR 1 day - 1 month) in early-stage v. 1 month (IQR 1 week - 1 month) in locally advanced disease, and $75.0 \%$ of all study participants attended within 1 month (early stage $79.7 \%$, locally advanced $72.1 \%$ ).

The relationship of each part of the delay to stage of disease is indicated in Fig. 1, with total delay for comparison. It is evident that, while there is a person-by-person variation in timeline to care, patients with early stage-disease tended to attend each part of their pathway more timeously. The most notable difference between patients with early and locally advanced disease was in time to acknowledging a symptom, whereas time to healthcare services and time to specialist care were similar (Fig. 1, A, B and C, respectively).

Similar to the analysis by stage, there were few patient characteristics associated with increased total delay; only travel time to hospital was found to be statistically significant $(p<0.001)$. When delay was separated into its three constituents, lack of internet access was associated with delay in acknowledging breast symptoms $(p=0.051$ ). Having someone in the family with breast cancer was also associated with a delay of $>6$ months in acknowledging breast symptoms ( $p=0.028)$. Fear of missing work due to appointments was associated with delay in accessing any healthcare services $(p=0.04)$. This delay in accessing healthcare services was also significantly more likely in patients with a low education (up to Grade 7) ( $p=0.008$ ).

\section{Discussion}

This study was designed to evaluate the pre-diagnostic timeline of patients newly diagnosed with breast cancer in an urban specialist breast clinic. We aimed to determine the relationship between delay to definitive breast cancer diagnosis, stage of disease at presentation and the socioeconomic characteristics that have previously been described as risk factors for delay to care in other resource-limited settings. We expected to reproduce the findings that delay was related to stage, and that both delay and stage would be related to some of the 
Table 1. Patient characteristics by stage of disease

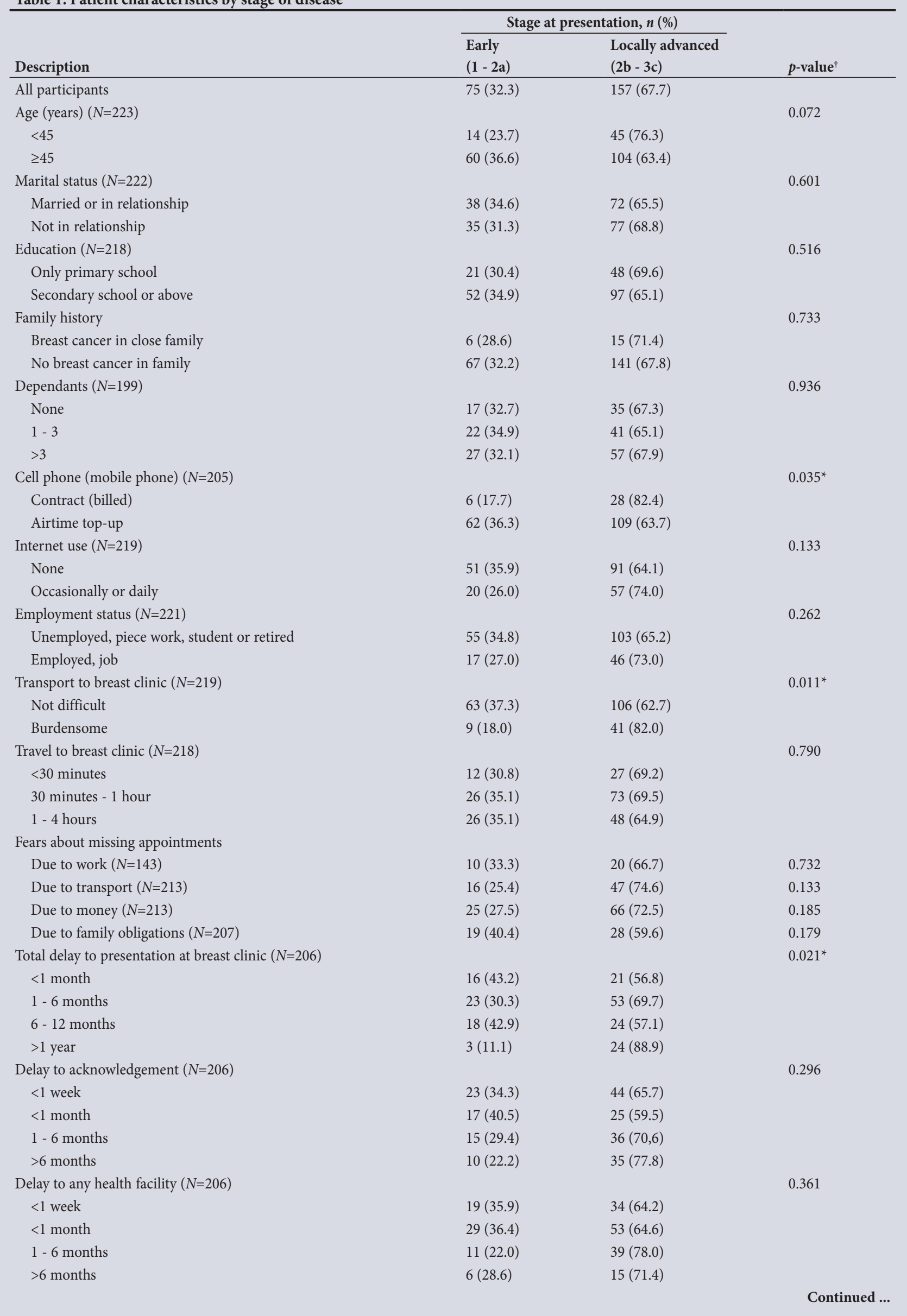


Table 1. (continued) Patient characteristics by stage of disease

\begin{tabular}{|c|c|c|c|}
\hline \multirow[b]{2}{*}{ Description } & \multicolumn{2}{|c|}{ Stage at presentation, $n(\%)$} & \multirow[b]{2}{*}{$p$-value } \\
\hline & $\begin{array}{l}\text { Early } \\
(1-2 a)\end{array}$ & $\begin{array}{l}\text { Locally advanced } \\
(2 b-3 c)\end{array}$ & \\
\hline Delay to specialist breast clinic $(N=206)$ & & & 0.257 \\
\hline$<1$ week & $34(34.0)$ & $66(66.0)$ & \\
\hline$<1$ month & $21(35.0)$ & $39(65.0)$ & \\
\hline $1-6$ months & $12(32.4)$ & $25(67.6)$ & \\
\hline$>6$ months & $2(11.1)$ & $16(88.9)$ & \\
\hline
\end{tabular}

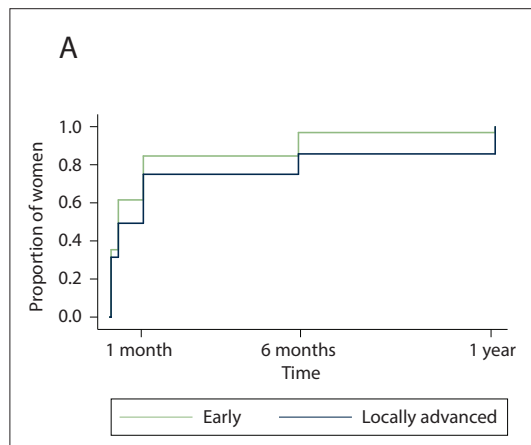

\section{B}

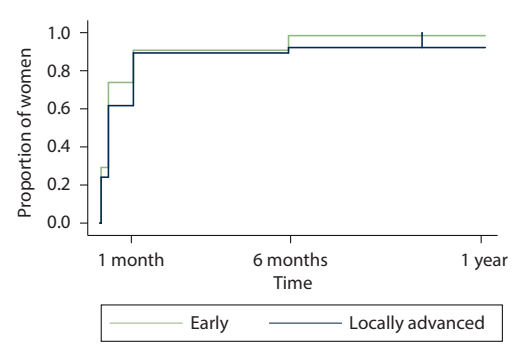

C

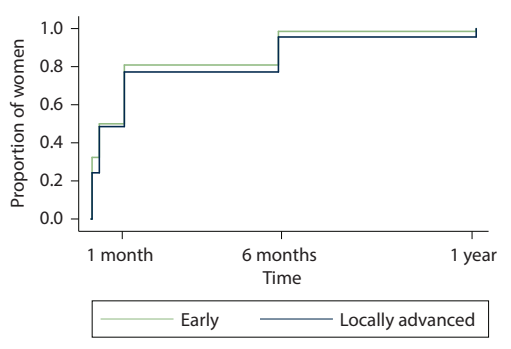

\section{D}

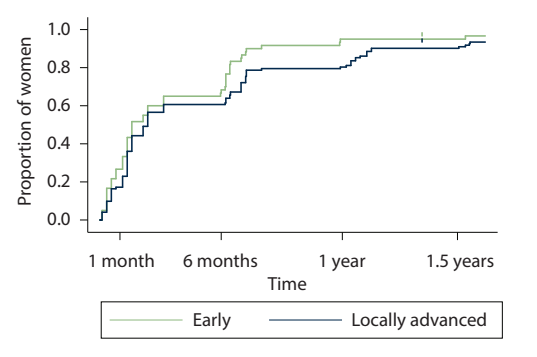

Fig. 1. Delay to presentation in patients with early and locally advanced disease: $A=$ time to acknowledging a symptom; $B=$ time from acknowledgement to any heath facility; $C=$ time from any health facility to specialist care; $D=$ total time from symptom to specialist care.

common risk factors for inequitable access to care seen in our environment, namely low income, low education, insecure job circumstances and poor access to transport and money. The significant relationship of a cell phone contract (as opposed to topup, indicating a more financially secure population) with locally advanced disease was an unexpected finding that requires more investigation.

In this study, a heterogeneous group of newly diagnosed participants was identified with few characteristics that defined them as at risk of presenting with locally advanced disease. However, it is worth pointing out some of the characteristics that make breast cancer a particular problem in our environment. More than a quarter of the newly diagnosed patients were aged $<45$ years. This has been a consistent find- ing in breast cancer studies in $\mathrm{SA}^{[2,18]}$ and may be a result of bias due to a younger overall population in SA, a younger population in this urban sample, or absence in this SA sample of the more biologically indolent cancers detected by screening older women in high-income settings. This is a younger cohort of women at risk of the disease than may be expected by healthcare practitioners, who therefore might not consider a breast cancer diagnosis possible or likely. Studies of provider-associated delays cite a lack of knowledge of breast cancer symptoms among providers, with inappropriate reassurance $e^{[10,19,20]}$ that could delay onward referral, specifically in younger patients.

Two-thirds of our patients presented with a locally advanced breast cancer (stage 2a 3c) with disease in both the breast and axilla, with a high risk of systemic micrometastatic disease. Almost all these patients require multimodality therapy, with chemotherapy, surgery and radiation all indicated. ${ }^{[21]}$ This requirement for additional therapies highlights the fact that late presentation in breast cancer is a crisis not only for the patient but also for the healthcare system. The earlier the cancer is diagnosed, the less likely it is that expensive and heavily underresourced therapies such as radiation will be required. One-quarter of all our patients presented with a tumour that was ulcerating or fixed to the skin and surrounding tissues, but there was no relationship between this finding and any self-reported delay.

Many studies in sub-Saharan Africa have described significant delays to presentation. ${ }^{[6,10,13,19,22-25]}$ In the present study, the average time to presentation at the breast clinic was 2 months from the time of noticing any symptoms, which is shorter than in many other African studies, where delay can be measured in years, but comparable to study groups from urban populations in other upper-middle-income countries worldwide. In Malaysia, $56 \%$ of patients presented over 3 months from the time of noticing symptoms; ${ }^{[26]}$ in Brazil this figure was $70 \%{ }^{[27]}$ and in Mexico it was $73.7 \% .{ }^{[28]}$ The only outlier in this group was in Thailand, ${ }^{[29]}$ where only $17 \%$ of patients presented after 3 months. However, this study of tertiary facilities in northern Thailand showed that $88 \%$ of all patients had a first consultation with a doctor, and for $89 \%$ of all patients this was in a hospital, thereby lessening the patient-related access issues of multiple visits, which can slow the progress of a patient through many tiers of the healthcare service towards tertiary or definitive care. ${ }^{[14]}$ In our study, the least delay in both early and locally advanced patients was seen between attending any healthcare services and attending the specialist clinic, with $>75.0 \%$ seen within 1 month, which confirms the improved accessibility for patients of open-access care and its contribution in 
preventing delay. However, this improved access is dependent on community knowledge of the facility and highlights the importance of community education and awareness, not just of breast symptoms but of the local facilities equipped to manage them.

Whether our results from an urban tertiary centre are generalisable more widely in SA, particularly in more rural areas, is uncertain. SA is becoming increasingly urbanised, and the populations in urban centres are growing. These populations tend to be young and may share many of the characteristics of our study population. There is access to tertiary breast cancer services in most of the main cities in $\mathrm{SA}$, and our findings can therefore inform other clinicians regarding the characteristics of their patients, and directions for future research around access to breast cancer care. They can also highlight to government the importance of a multimodal method of improving cancer care - addressing education and awareness, but also opening up access to care to facilitate early diagnosis.

\section{Study limitations}

The limitations of this study should be acknowledged. Many patients with breast cancer may not present until the very latest stages, where palliation is appropriate and up-referral for interventional therapy is decided against. Lack of a comprehensive screening programme also means that most breast cancer is detected after a clinical symptom, which will predispose toward a later stage. There may be women, even in urban environs, who never reach the specialist clinic despite open-access facilities. It is notoriously hard to characterise these women, but it can only be assumed that they are more likely to have late-stage disease (indeed end-stage disease) and have the longest time to presentation. This is an important source of selection bias in this current study, but one that is difficult to overcome. Additionally, some women may not accurately remember when they first acknowledged symptoms, or the time taken to attend for care. This would be likely to affect all women equally, however, and therefore not attract bias on the comparison of stage and time to presentation.

\section{Conclusions}

Despite an increase in breast cancer awareness in SA and the recent national Breast Cancer Prevention and Control Policy, two-thirds of our patients presented with locally advanced breast cancer. While women with transport problems and work fears were more likely to present with locally advanced disease, there were few socioeconomic characteristics that could identify those at risk of delay to presentation. Most women delayed longer than recommended in both seeking any care and attending specialist breast care. Although time to acknowledge a symptom was associated with the most delay, the reduction in time to specialist care in these circumstances supports the availability of open-access care to facilitate access to care once symptoms are recognised.

Declaration. This article was part of SR's $\mathrm{PhD}$ thesis.

Acknowledgements. The authors acknowledge the invaluable partnership of the participants and their families as well as the assistance of the clinic staff in enabling this research to be carried out. The authors would be happy to share all the primary and supplementary data with any interested parties within the boundaries of ethical approval.

Author contributions. SR conceived and designed the study, collected and interpreted data, drafted the manuscript and approved the final version.
KS and DK analysed and interpreted data, critically revised the manuscript and approved the final version. $\mathrm{CF}$ and $\mathrm{C}-\mathrm{AB}$ interpreted data, critically revised the manuscript and approved the final version

Funding. None.

Conflicts of interest. None.

1. Statistics South Africa. Mortality and causes of death in South Africa, 2015: Findings from death notification. 2017. http://www.statssa.gov.za/publications/P03093/P030932015.pdf (accessed 1 May

notifica

South African National Cancer Registry. Summary statistics of cancer diagnosed histologically in 2012. 2012. http://www.nioh.ac.za/wp-content/uploads/2018/03/NCR-2012-results.pdf (accessed 1 May 2018).

3. Allemani C, Weir HK, Carreira H, et al. Global surveillance of cancer survival 1995 - 2009: Analysis of individual data for 25676887 patients from 279 population-based registries in 67 countries (CONCORD-2). Lancet 2015;385(9972):977-1010. https://doi.org/10.1016/S0140-6736(14)62038-9

4. National Department of Health, South Africa. Breast Cancer Prevention and Control Policy. 2017. http:// www.health.gov.za/index.php/shortcodes/2015-03-29-10-42-47/2015-04-30-08-18-10/2015-04-30-0824-27 (accessed 1 May 2018).

5. National Department of Health, South Africa. Launch of cancer policies 2017. http://www.health.gov. za/index.php/gf-tb-program/361-launch-of-cancer-policies-2017 (accessed 15 September 2017).

6. Pruitt L, Mumuni T, Raikhel E, et al. Social barriers to diagnosis and treatment of breast cancer in patients presenting a teaching hospital in tbadan, Nigeria. Glob Public Health 2014;10(3):331-344 patients presenting at a teaching hospital in Ib
https://doi.org/10.1080/17441692.2014.974649

7. Ukwenya AY, Yusufu LM, Nmadu PT, Garba ES, Ahmed A. Delayed treatment of symptomatic breast Ukwenya AY, Yusufu LM, Nmadu PT, Garba ES, Ahmed A. Delayed treatment
cancer: The experience from Kaduna, Nigeria. S Afr J Surg 2008;46(4):106-110.

cancer: The experience from Kaduna, Nigeria. S Afr J Surg 2008;46(4):106-110.
8. Iskandarsyah A, de Klerk C, Suardi DR, Soemitro MP, Sadarjoen SS, Passchier J. Psychosocial and . Iskandarsyah A, de Klerk C, Suardi DR, Soemitro MP, Sadarjoen SS, Passchier J. Psychosocial and
cultural reasons for delay in seeking help and nonadherence to treatment in Indonesian women with breast cancer: A qualitative study. Health Psychol 2014;33(3):214-221. https://doi.org/10.1037/ a0031060

9. Grunfeld EA, Kohli N. Beliefs about breast cancer and help-seeking intentions for the disease among women in India. Women Health 2010;50(4):327-341. https://doi.org/10.1080/03630242.2010.498752

10. Salih AM, Alfaki MM, Alam-Elhuda DM. Factors delaying presentation of Sudanese breast cancer patients: An analysis using Andersen's model. Asian Pac J Cancer Prev 2016;17(4):2105-2110. https:// doi.org/10.7314/APJCP.2016.17.4.2105

1. Ajekigbe A. Fear of mastectomy: The most common factor responsible for late presentation of carcinoma of the breast in Nigeria. Clin Oncol (R Coll Radiol) 1991;3(2):78-80.

12. O’Brien KS, Soliman AS, Annan K, Lartey RN, Awuah B, Merajver SD. Traditional herbalists and cancer management in Kumasi, Ghana. J Cancer Educ 2012;27(3):573-579. https://doi.org/10.1007/ s13187-012-0370-z

13. Gabrielly A, Freitas Q, Weller M. Patient delays and system delays in breast cancer treatment in developed and developing countries. Cien Saude Colet 2015;20(10):3177-3189. https://doi. org/10.1590/1413-812320152010.19692014

14. Rayne S, Lince-Deroche N, Hendrickson C, et al. Characterizing breast conditions at an open-access breast clinic in South Africa: A model that is more than cancer care for a resource-limited setting. BMC Health Serv Res 2017;17(63):1-10. https://doi.org/10.1186/s12913-016-1959-4

15. Rayne S, Schnippel K, Wright K, Kruger D, Firnhaber C, Benn C. Fear of treatments surpasse demographic and socioeconomic factors in affecting patients with breast cancer in urban South Africa. J Glob Oncol 2016;3(2):125-134. https://doi.org/10.1200/JGO.2015.002691

16. Harris PA, Taylor R, Thielke R, Payne J, Gonzalez N, Conde JG. Research electronic data capture (REDCap): A metadata-driven methodology and workflow process for providing translational research informatics support. J Biomed Inform 2009;42(2):377-381. https://doi.org/10.1016/j.jbi.2008.08.010

17. Giuliano AE, Connolly JL, Edge SB, Mittendorf EA. Breast cancer - major changes in the American Joint Committee on Cancer Eighth Edition Cancer Staging Manual. CA Cancer J Clin 2017;67(4). https://doi.org/10.3322/caac.21393

18. Cubasch H, Joffe M, Hanisch R, et al. Breast cancer characteristics and HIV among 1,092 women in Soweto, South Africa. Breast Cancer Res Treat 2013;140(1):177-186.

19. Kohler RE, Gopal S, Miller AR, et al. A framework for improving early detection of breast cancer in Kohler RE, Gopal S, Miller AR, et al. A framework for improving early detection of breast cancer in
sub-Saharan Africa: A qualitative study of help-seeking behaviors among Malawian women. Patient sub-Saharan Africa: A qualitative study of help-seeking behaviors among M
Educ Couns 2017;100(1):167-173. https://doi.org/10.1016/j.pec.2016.08.012

20. McEwan J, Underwood C, Corbex M. 'Injustice! That is the cause': A qualitative study of the social, economic, and structural determinants of late diagnosis and treatment of breast cancer in Egypt. Cancer Nurs 2014;37(6):468-475. https://doi.org/10.1097/NCC.0000000000000118

21. Gradishar W, Salerno KE. NCCN Guidelines Update: Breast cancer. J Natl Compr Canc Netw 2016;14(5S):641-644. https://doi.org/10.6004/jnccn.2016.0181

22. Brinton L, Figueroa J, Adjei E, et al. Factors contributing to delays in diagnosis of breast cancers in Ghana, West Africa. Breast Cancer Res Treat 2017;162(1):105-114. https://doi.org/10.1007/s10549016-4088-1

23. Opoku SY, Benwell M, Yarney J. Knowledge, attitudes, beliefs, behaviour and breast cancer screening practices in Ghana, West Africa. Pan Afr Med J 2012;11:28.

24. Clegg-Lamptey J, Dakubo J, Attobra YN. Why do breast cancer patients report late or abscond during treatment in Ghana? A pilot study. Ghana Med J 2009;43(3):127-131.

25. Galukande $\mathrm{M}$, Wabinga $\mathrm{H}$, Mirembe $\mathrm{F}$. Breast cancer survival experiences at a tertiary hospital in sub-Saharan Africa: A cohort study. World J Surg Oncol 2015;13:220. https://doi.org/10.1186/s12957015-0632-4

26. Lim JNW, Potrata B, Ng C, et al. Barriers to early presentation of self-discovered breast cancer in Singapore and Malaysia: A qualitative multicentre study. BMJ Open 2015;5(12):e009863. https://doi. org/10.1136/bmjopen-2015-009863

27. Barros AF, Uemura G, Soares JL. Time to access breast cancer treatment in the Federal District, Central Brazil. Braz J Gynecol Obstet 2013;35(10):1-9. https://doi.org/10.1590/S0100-72032013001000006

28. Unger-Saldaña $\mathrm{K}$. Challenges to the early diagnosis and treatment of breast cancer in developing countries. World J Clin Oncol 2014;5(3):465-478. https://doi.org/10.5306/wjco.v5.i3.465

29. Poum A, Promthet S, Duffy SW, Parkin DM. Factors associated with delayed diagnosis of breast cancer in Northeast Thailand. J Epidemiol 2014;24(2):102-108. https://doi.org/10.2188/jea.JE20130090 